\title{
SURVEY ON SENSOR PROTOCOL FOR INFORMATION VIA NEGOTIATION (SPIN) PROTOCOL
}

\author{
Vidhi S. Patel ${ }^{1}$, Chandresh R. Parekh ${ }^{2}$ \\ ${ }^{1}$ Wireless and mobile Computing GTU-PG School (BISAG), Gandhinagar \\ ${ }^{2}$ Department of Electronics and communication, Government Engineering College, Gandhinagar
}

\begin{abstract}
Wireless sensor network is a collection of sensor nodes which sense application specific data and send it to sink to perform some task. One of the major issues due to energy constraint in wireless sensor network is data transmission. Many routing protocols till day have been proposed to route data efficiently in order to increase network lifetime. Sensor Protocol for Information via Negotiation (SPIN) is one of the most popular data centric dissemination protocols. It efficiently disseminates data among other nodes in the network. This protocol uses meta-data for negotiation and eliminates the transmission of the outmoded data throughout the network. This paper survey issues in SPIN protocol and explain about possible enhancement of SPIN protocol.
\end{abstract}

Keywords: Wireless Sensor Network, Sensor Protocol via Information Negotiation, Advertisement Message, Request Message

$* * *$

\section{INTRODUCTION}

Wireless sensor network (WSN) refers to a group of spatially dispersed and dedicated sensors for monitoring and recording the physical conditions of the environment and organizing the collected data at a central location. WSNs measure environmental conditions like temperature, sound, pollution levels, humidity, wind speed and direction, pressure, etc.WSNs were initially designed to facilitate military operations but its application has since been extended to health, traffic, and many other consumer and industrial areas.

A WSN consists of anywhere from a few hundreds to thousands of sensor nodes. The sensor node equipment includes a radio transceiver along with an antenna, a microcontroller, an interfacing electronic circuit, and an energy source, usually a battery. The size of the sensor nodes can also range from the size of a shoe box to as small as the size of a grain of dust. As such, their prices also vary from a few cents to hundreds of dollars depending on the functionality parameters of a sensor like energy consumption, computational speed rate, bandwidth, and memory.

WSN has a challenging issue of having good routing protocol having less memory usage and maximum throughput out of it. But existing protocols are not $100 \%$ fulfill these requirements so we need to expand the working protocols and need to improve their performance.

\section{SPIN PROTOCOL}

(SPIN) as name suggest is a negotiation based protocol and is among the early work to pursue a data-centric routing mechanism. The idea behind SPIN is to name the data using high level descriptors or meta-data. Before transmission, metadata are exchanged among sensors via data advertisement mechanism, which is the key feature of SPIN. Each node upon receiving new data, advertises it to its neighbors and interested neighbors, i.e. those who do not have the data, retrieve the data by sending a request message. SPIN's meta-data negotiation solves the classic problems of flooding such as redundant information passing, overlapping of sensing areas and resource blindness thus, achieving a lot of energy efficiency. There are three messages defined in SPIN to exchange data between nodes. These are: ADV message to allow a sensor to advertise a particular meta-data, REQ message to request the specific data and DATA message that carry the actual data. Below figure redrawn from, summarizes the steps of the SPIN protocol.

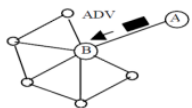

(a)

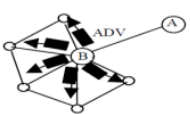

(d)

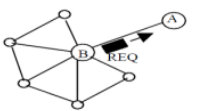

(b)

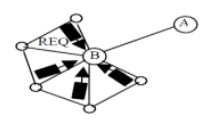

(e)

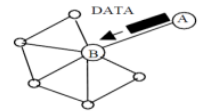

(c)

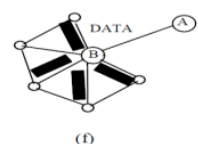

Fig 1 SPIN Protocol Node A starts by advertising its data to node B [1] 
(a) Node $\mathrm{B}$ responds by sending a request to node $\mathrm{A}$

(b) After receiving the requested data

(c) Node B then sends out advertisements to its neighbours

(d) Who in turn send requests back to B (e-f).

One of the advantages of SPIN is that topological changes are localized since each node needs to know only its single-hop neighbours. SPIN gives a factor of 3.5 less than flooding in terms of energy dissipation and meta-data negotiation almost halves the redundant data.

However, SPIN's data advertisement mechanism cannot guarantee the delivery of data. For instance, if the nodes that are interested in the data are far away from the source node and the nodes between source and destination are not interested in that data, such data will not be delivered to the destination at all.

Therefore, SPIN is not a good choice for applications such as intrusion detection, which require reliable delivery of data packets over regular intervals.

\section{PROBLEM STATEMENT}

As survey made on SPIN protocol, by reading different literatures and researches made before, the SPIN protocol still have some loop falls that need to be improve. Papers referred says different modifications made on SPIN protocol but all improvements have some drawback and that need to be solve.

1) One improvement made in SPIN is M-SPIN. That is Shown in bellow figure.

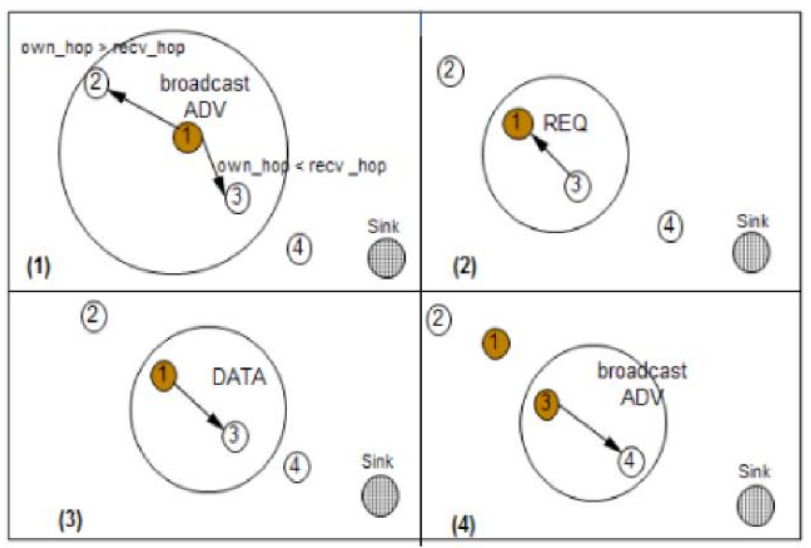

Fig. 2 Transmission in M-SPIN Protocol[5]

(1) Node 1 starts advertising its data to all of its neighbors.

(2) Node 3 responds by sending a request to node 1 .

(3) After receiving the request, node 1 sends the data.

(4) Node 3 again sends advertisement out to its neighbors for the data that it received from node-1.[5]

M-SPIN protocol which is more energy efficient rather than traditional SPIN, but problem in that is, this protocol leads to one problem that is nodes nearby sink node dies early, so as per that paper it is one criteria of research to make that nodes power efficient or can make whole network power aware by assuming mobile environment in WSN.

2) Another idea is of SPIN-pi protocol. In traditional SPIN protocol If a node's neighbors are not forwarding data, the data will not continue to be forwarded, so that the user couldn't receive the information. So solution of that is in SPIN-pi that is adding plug-in nodes in the SPIN protocol. They have used nodes which are have AC Power supply, if according to application if there is requirement of having deployments of node in some forest or under water where no continous powwer supply can be made, we can use solar cell plugged node, which can takes continuous energy from sun light. Below figure shows an example of SPIN-pi protocol.

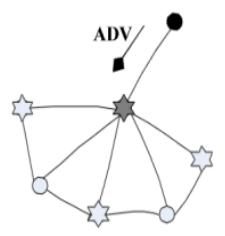

(a)

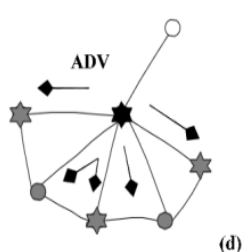

(d)

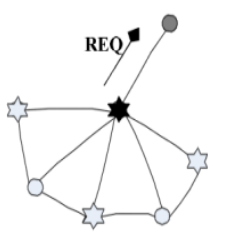

(b)

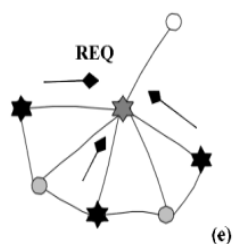

(e)

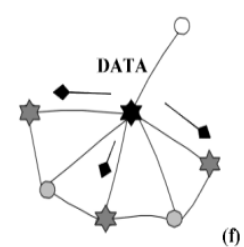

Ps Plug-in node

Battery consumed node
Fig. 3 The network communication process in SPIN-Pi[6]

So, by getting idea of such more power efficient nodes and deploying more amount of such much power efficient nodes in network we can improve the life time of our network and make it more reliable and efficient.

3) In this paper they have introduced new protocol named SPIN- I. In SPIN protocol there are problem of "blind forward" and "data inaccessible". To avoid these problem this protocol using three way handshake mechanism:

1) Data broadcasting stage

2) Data requesting stage

3) Data transmission phase.

In SPIN-I they include timer concept in broadcasting stage means at time of advertisement. And at receiver side means a node who receives ADV message includes flag to determine its energy level.[6] 
They simulated SPIN and SPIN-I protocols under certain idealized environment and in that environment SPIN-I performed better than SPIN.

SPIN-I protocol is good solution to solve the "blind forward" and "data unreachable" problem. However, because here the SPIN-I protocol is simulated in a more idealized situation, and its transmission time is longer than SPIN, the solution of these problems needs further research.

\section{PROPOSED SOLUTION}

According to above literatures, here defined problem statement as "Reliable data transmission in sensor network using M-SPIN protocol and deploying plug-in nodes in between."

In such proposed scheme sensor node would be arranged in cellular manners. Means at center of each cell there is one plugin node and around that plug-in node there are simple sensor nodes. This plug-in node concept is taken from literature discussed 2.6[6], and routing protocol M-SPIN would be used.

There are some pre-assumption that would be considered. They are, assuming that all the nodes used in such network design are homogeneous, but there is difference between nodes in terms of their energy efficiency. That is there are normal sensor nodes which are traditional sensor nodes works on dry battery cells where as other nodes called plug-in nodes are nodes which uses solar energy so they are more energy efficient than that normal sensor nodes.

Also assume that the energy efficiency of plug-in node is high then normal sensor nodes so their range of the transmission would also high than other normal nodes, and plug-in nodes are high energy efficient so that they can route data for long time of period because they are continuously getting power supply and also they never drop any data packet passed through it. Plug-in node always route packet to next destination without failure and if any predecessor node of plug-in node fail to transmit data then plug-in node are capable to retransmit data. So it is reliable to having concept of acknowledgment at plug-in nodes.

As per the cellular arrangements of nodes, each sensor node is directly connected to minimum 3 plug-in nodes. So in path of routing there are minimum half of the node would be plug-in nodes. So it improves the reliability of path. If we assume that each plug-in node can have more range than other sensor nodes then there might be possibility to send data from one plug-in node to another plug-in node if any in between node would fail or not send data ahead that would improve reliability and save energy too. In below figure it shows the proposed network structure.

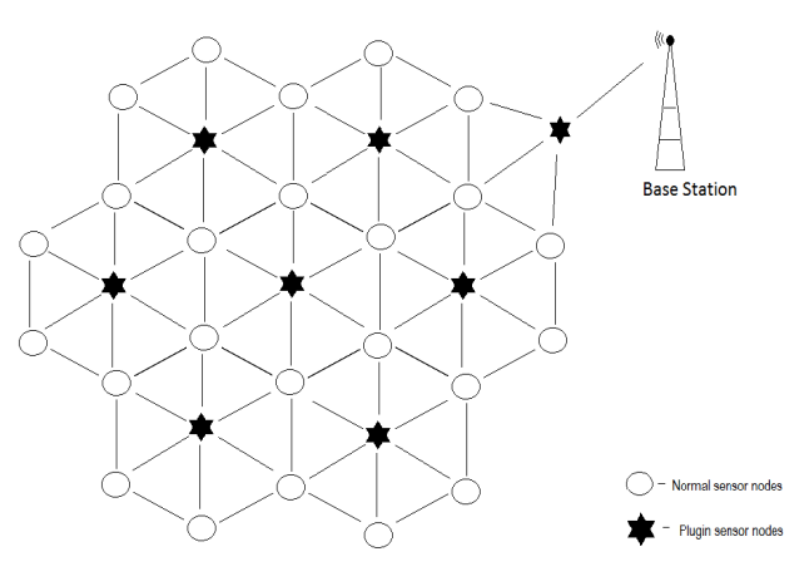

Fig. 4 Proposed solution's network topology

In proposed scheme routing would be done according to the MSPIN protocol. In algorithm of the routing scheme of this improved protocol First stage is Distance discovery, then Negotiation and last stage is Data Transmission.

So, it works similarly to M-SPIN mechanism but difference is that, problem in M-SPIN was the node near the Base Station drain out first, so optimizing that problem using plug-in node here. Because plug-in nodes uses solar cells as battery backup so it would charge regularly and other problem in SPIN protocol is reliability, so by adding plug-in nodes in between they improve network efficiency rather than normal nodes because the average failure of network minimized by their installation.

Algorithm: If we consider node A wants to send data to the Base station (sink) node.

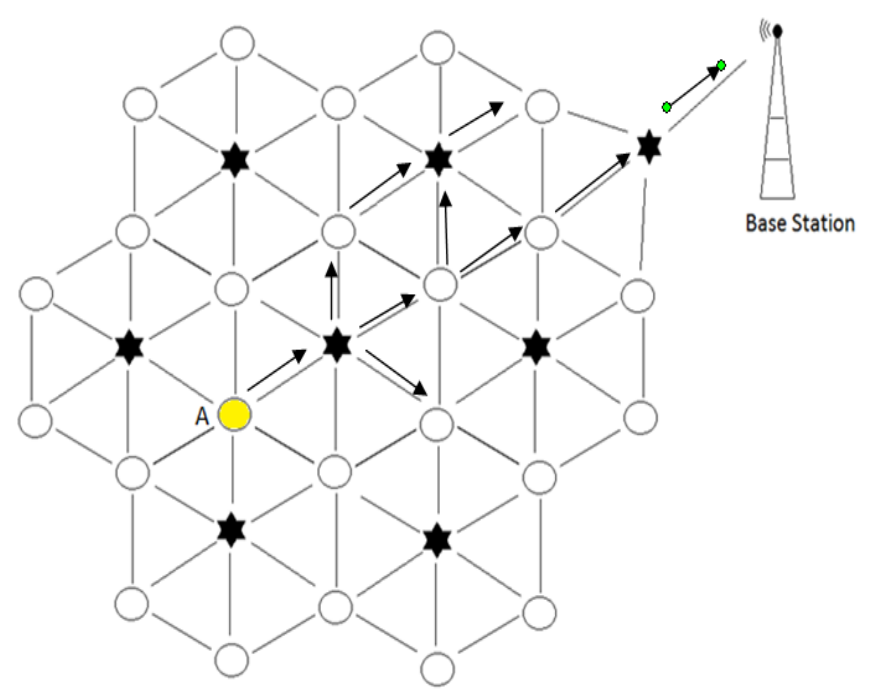

Fig. 5 Algorithm illustration 
Step1: Initially Base station (Sink node) sends the Startup packet in the network. Startup packet containing three values, Type, Node ID and Hop. Type means message type, Node ID means ID of sender node and hop means the hop distance of node from sink node.

Step 2: After the distance discovery phase complete, it starts negotiation phase. Same as SPIN-BC protocol it sends ADV message to its neighbor and the neighbor whose hop distance is lesser than sender of ADV only can send REQ message. Here A is the sender node. Who sends ADV message but the neighbor whose hop distance is lesser than hop distance of A only can send REQ message.

Step 3: After sender node get the REQ message it comes to data transmission mode. In which sender sends actual data to that link. This negotiation and Data transmission phase would repeat until it reaches to Base Station.

To increase reliability we can add acknowledgement feature at plug-in node to make confidentiality of data transfer.

\section{CONCLUSIONS}

The WSN is different than other wired or wireless networks like MANET or VANET. The main crucial issue in WSN is minimum amount of energy. Though WSN is made up of hundreds or thousands of nodes which are stand alone and communicate to each other using hope by hope data transmission in network and having minimum amount of energy. So if any node dies because of energy drain then the link made up of that node would affected and it causes network failure after more number of node dies. So, to make network more reliable and increase its throughput the only option we have is to make any WSN power aware. Here in SPIN protocol using different techniques and aspects researches happened and still there are some drawbacks remain. To make network reliable and improve network lifetime proposed given solution is being implemented in future work. Inspired by the previous derived solutions this proposed solution would be effective for network improvement and it would help network to.

\section{ACKNOWLEDGMENTS}

Warmly thanks to God, my family and friends to give me such a great support and help for carry out this work. And lots of thanks to large number of open sources and completed projects from which got so much of inspiration and ideas to do this work.

\section{REFERENCES}

[1] Routing techniques in wireless sensor networks: a survey- jamal n. al-karaki, the hashemite universityahmed e. kamal, iowa state university

[2] Simulation Study For Routing Protocols In Sensor Networks- AbdelazizAraar, Hakim Khali, and Riyadh A. MehdiFaculty of Computer Sciences, Ajman University, Ajman, UAE
The Analysis And Research OfWsn's Energy efficient Routing Protocols- Jie XU, Fanglu NING, Dawei JIANG*Eastern Liaoning University, Dandong, China

[4] Comparison and Analysis Data-Centric Routing protocols in wireless sensor networks- Zahra GhaffariDepartment of ComputerEngineering and InformationTechnology, mirkabir University Tehran, Iran, TaliehJafari,Department of Computer Engineeringand Information Technology,Amirkabir University Tehran, Iran, HosseinEskandariShahraki,Department of Computer Engineeringand Information Technology,Amirkabir University Tehran, Iran

[5] A Modified SPIN for Wireless Sensor NetworksZeenatRehenaSchool of Mobile Computing andCommunication, JadavpurUniversityKolkata - 700 098, India, Sarbani Roy Dept of Computer Science \&Engineering, JadavpurUniversityKolkata - 700 032, India, NandiniMukherjeeDept of Computer Science \&Engineering, JadavpurUniversityKolkata - 700 032, India-2011

[6] Improving Routing Protocols of WSN in WHSNRuochen Tan, Yang Xu, Zheng ChenUniversity of Electronic Science and Technology of ChinaChengdu 611731, P.R.China, Min Zha, Tina TsouHuawei Technologies Co., Ltd.Shenzhen 518129,P.R.China2010

[7] Energy Saving Routing Algorithm Based on SPIN Protocol in WSN-Luwei Jing, and FengLiuCollege of Science, Huazhong Agricultural University, Wuhan, 430070, Chin, YulingLiCollege of Information Engineering, Chutian University of Huazhong Agricultural University, Wuhan, 430205, China-2011

[8] SPIN Implementation in TinyOS Environment usingnesC- Zeenat Rehena1, Krishanu Kumar2, Sarbani Roy2, Nandini Mukherjee21School of Mobile Computing and Communication, Jadavpur University2 Department of Computer Science \& Engineering, JadavpurUniversityKolkata - 700 032, India

[9] Combining SPIN with ns-2 for protocol OptimizationPedro Merino1 and Alberto Salmeron1 University of Malaga, Campus de Teatinos, 29071, Malaga, Spain fpedro, salmerong@1cc.uma.es

[10] Negotiation based protocol for Disseminating Information in Wireless Sensor Networks- Joanna Kulik, Wendi RabinerHeinzelman, and hariBalakrishnanMassachusetta Institute of Technology, Cambridge, MA 02139

[11] Performance Comparison of SPIN basedPush-Pull Protocols,RavishKhosla, XuanZhong, GunjanKhanna, SaurabhBagchi, and Edward J. CoyleSchool of Electrical and Computer EngineeringPurdue University, West Lafayette, Indiana 47907 